1. Bernstein, I.D., Andrews, R.G., and Rowley, S. 1994 Isolation of human hematopoietic stem cells. Blood Cells. 20:15-24.

2. Tsai, R.J., Li, L.M., and Chen, J.K. 2000. Reconstruction of damaged corneas by transplantation of autologous limbal epithelial cells. N. Engl. J. Med. 343:86-93.

3. Stenn, K.S., and Cotsarelis, G. 2005. Bioengineering the hair follicle: fringe benefits of stem cell technology. Curr. Opin. Biotechnol. 16:493-497.

4. Morris, R.J. 2000. Keratinocyte stem cells: targets for cutaneous carcinogens. J. Clin. Invest. 106:3-8.

5. Cotsarelis, G., Sun, T.T., and Lavker, R.M. 1990 Label-retaining cells reside in the bulge area of pilosebaceous unit: implications for follicular stem cells, hair cycle, and skin carcinogenesis. Cell. 61:1329-1337.

6. Morris, R.J., et al. 2004. Capturing and profiling adult hair follicle stem cells. Nat. Biotechnol. 22:411-417.

7. Oshima, H., Rochat, A., Kedzia, C., Kobayashi, K., and Barrandon, Y. 2001. Morphogenesis and renewal of hair follicles from adult multipotent stem cells. Cell. 104:233-245.

8. Tumbar, T., et al. 2004. Defining the epithelial stem cell niche in skin. Science. 303:359-363.

9. Lyle, S., et al. 1998. The C8/144B monoclonal antibody recognizes cytokeratin 15 and defines the location of human hair follicle stem cells. J. Cell Sci. 111:3179-3188.

10. Ohyama, M., et al. 2006. Characterization and isolation of stem cell-enriched human hair follicle bulge cells. J. Clin. Invest. 116:249-260. doi:10.1172/ JCI26043.

11. Liu, Y., Lyle, S., Yang, Z., and Cotsarelis, G. 2003. Keratin 15 promoter targets putative epithelial stem cells in the hair follicle bulge. J. Invest. Dermatol. 121:963-968.

12. Ito, M., et al. 2005. Stem cells in the hair follicle bulge contribute to wound healing but not to homeostasis of the epidermis. Nat. Med. 11:1351-1354.

13. Trempus, C., et al. 2003. Enrichment for living murine keratinocytes from the hair follicle bulge with the cell surface marker CD34. J. Invest. Dermatol. 120:501-511.

14. Nishimura, E.K., et al. 2002. Dominant role of the niche in melanocyte stem-cell fate determination. Nature. 416:854-860.

15. Ito, M., Kizawa, K., Toyoda, M., and Morohashi, M. 2002. Label-retaining cells in the bulge region are directed to cell death after plucking, followed by healing from the surviving hair germ. J. Invest. Dermatol. 119:1310-1316.

16. Gilliam, A.C., et al. 1998. The human hair follicle: a reservoir of CD40+ B7-deficient Langerhans cells that repopulate epidermis after UVB exposure. J. Invest. Dermatol. 110:422-427.

17. Kumamoto, T., et al. 2003. Hair follicles serve as local reservoirs of skin mast cell precursors. Blood. 102:1654-1660.

18. Rosenblum, M.D., et al. 2004. Expression of CD200 on epithelial cells of the murine hair follicle: a role in tissue-specific immune tolerance? J. Invest. Dermatol. 123:880-887.

\title{
Delving deeper into MALT lymphoma biology
}

\section{Francesco Bertoni and Emanuele Zucca}

Laboratory of Experimental Oncology and Lymphoma Unit, Oncology Institute of Southern Switzerland, Bellinzona, Switzerland.

\begin{abstract}
Mucosa-associated lymphoid tissue (MALT) lymphomas can arise in a variety of extranodal sites. Interestingly, at least 3 different, apparently site-specific, chromosomal translocations, all affecting the NF- $\kappa B$ pathway, have been implicated in the development and progression of MALT lymphoma. The most common is the translocation $t(11 ; 18)(q 21 ; q 21)$, which results in a fusion of the cIAP2 region on chromosome $11 \mathrm{q} 21$ with the MALT1 gene on chromosome 18q21 and is present in more than onethird of cases. The frequency of this translocation is site-related: common in the gastrointestinal tract and lung, rare in conjunctiva and orbit, and almost absent in salivary glands, thyroid, liver, and skin. In this issue of the JCI, Hu et al. add to our understanding of the molecular consequences of this translocation, showing that its fusion product, cIAP2MALT1, may concomitantly contribute to lymphomagenesis both as a tumor suppressor gene and as an oncogene (see the related article beginning on page 174).
\end{abstract}

\section{MALT Iymphoma: \\ the clinical background}

Extranodal marginal zone B cell lymphoma (EMZL) of mucosa-associated lymphoid tissue (MALT), also known as MALT lymphoma, is a neoplastic disease that constitutes approximately $8 \%$ of all nonHodgkin lymphomas (1-4). It can arise in a variety of extranodal sites and occurs

Nonstandard abbreviations used: BIR, baculoviral IAP repeat; CARD, caspase recruitment domain; cIAP2, cellular inhibitor of apoptosis protein 2 ; DD, death domain; EMZL, extranodal marginal zone B cell lymphoma; IAP, inhibitor of apoptosis protein; IкB, inhibitory $\kappa \mathrm{B}$; IKK, IкB kinase; MALT, mucosa-associated lymphoid tissue; NES, nuclear export signal.

Conflict of interest: The authors have declared that no conflict of interest exists.

Citation for this article: J. Clin. Invest. 116:22-26 (2006). doi:10.1172/JCI27476. most often in organs such as the stomach, the salivary glands, or the thyroid, where lymphocytes are normally absent. MALT is the result of chronic phlogistic events in response to either infectious conditions such as Helicobacter pylori-associated chronic gastritis, or autoimmune disorders like Hashimoto thyroiditis and myoepithelial sialadenitis. In these conditions abnormal B cell clones can progressively replace the normal B cell population of the inflammatory tissue, giving rise to the EMZL. Over 10 years ago, $H$. pylori was identified as an etiologic factor in gastric MALT lymphomas after the demonstration of tumor regression in the majority of early-stage cases treated with anti-Helicobacter antibiotic therapy, and this tumor therefore became a popular model of the close pathogenetic link between chronic inflammation and lymphoma development. Other bacterial infections were later possibly implicated in the pathogenesis of MALT lymphomas arising in the skin (Borrelia burgdorferi), in the ocular adnexa (Chlamydia psittaci), and in the small intestine (Campylobacter jejuni) $(1,4)$.

The prognosis for patients with MALT lymphomas is good; these tumors usually have an indolent course with overall 5-year survival rates greater than $80 \%$, but rare cases with histologic transformation in cases of aggressive diffuse large-cell lymphoma have been described. It is nowadays generally accepted that eradication of $H$. pylori with antibiotics should be used as the sole initial treatment of localized gastric MALT lymphoma, while the use of anti-infectious treatment in nongastric locations is still under investigation. Other effective treatment approaches include radiotherapy, chemotherapy, and antiCD20 mAbs $(2,3)$.

\section{Many chromosomal translocations affecting the same pathway}

Four main recurrent chromosomal translocations have been associated with the pathogenesis of EMZLs: $t(11 ; 18)(\mathrm{q} 21 ; \mathrm{q} 21)$, $\mathrm{t}(1 ; 14)(\mathrm{p} 22 ; \mathrm{q} 32), \mathrm{t}(14 ; 18)(\mathrm{q} 32 ; \mathrm{q} 21)$, and $\mathrm{t}(3 ; 14)(\mathrm{p} 14.1 ; \mathrm{q} 32)(5-8)$ (Table 1). The latter is the most recently described and establishes the juxtaposition of the transcription factor FOXP1 next to the enhancer region 


\section{Table 1}

Clinical and biological features associated with the 4 main recurrent chromosomal translocations described in MALT lymphomas

\begin{tabular}{|c|c|c|c|c|}
\hline & $t(11 ; 18)(q 21 ; q 21)$ & $t(1 ; 14)(p 22 ; q 32)$ & $t(14 ; 18)(q 32 ; q 21)$ & $t(3 ; 14)(p 14.1 ; q 32)$ \\
\hline Product & cIAP2-MALT1 fusion protein & Overexpression of BCL10 & Overexpression of MALT1 & Overexpression of FOXP1 \\
\hline$\%$ of cases & $15-40 \%$ & $1-2 \%$ & $20 \%$ & Unknown \\
\hline Main Iymphoma localizations & Stomach, lung, intestine & Stomach, lung & $\begin{array}{l}\text { Salivary glands, skin, } \\
\text { ocular adnexa, liver, lung }\end{array}$ & $\begin{array}{l}\text { Thyroid, ocular } \\
\text { adnexa, skin }\end{array}$ \\
\hline MALT1 expression & Cytoplasmic, weak & Cytoplasmic, weak & Cytoplasmic, strong & Unknown \\
\hline BCL10 expression & Nuclear, strong & Nuclear, strong & Cytoplasmic, strong & Unknown \\
\hline F0XP1 expression & Unknown & Unknown & Unknown & Nuclear \\
\hline$N F-\kappa B$ activation & Yes & Yes & Yes & Unknown \\
\hline Additional genomic abnormalities & No & Yes & Yes & Yes \\
\hline Histologic transformation & No & Yes & Yes & Yes \\
\hline
\end{tabular}

of the Ig heavy chain genes (8); the pathogenetic relevance of this translocation is still unclear. Interestingly, the other 3 seemingly disparate translocation types appear to affect the same signaling pathway, resulting in the activation of NF- $\kappa B$, a transcription factor with a central role in immunity, inflammation, and apoptosis (1). The $\mathrm{t}(1 ; 14)(\mathrm{p} 22 ; \mathrm{q} 32)$ translocation is detected in only $1-2 \%$ of cases of EMZL. The translocation results in overexpression of the BCL10 gene due to the juxtaposition with the promoter region of the Ig heavy chain genes. The BCL10 gene (also known as CARMEN, CIPER, CLAP, $c-E 10$, and $m E 10$ ) codes for an adaptor protein implicated, as discussed below, in surface receptor signaling and highly expressed in the nucleus of the neoplastic B cells of EMZLs carrying this translocation $(6,9)$. The $t(14 ; 18)(\mathrm{q} 32 ; \mathrm{q} 21)$ translocation, described in approximately $20 \%$ of EMZL cases and cytogenetically identical to the $\mathrm{t}(14 ; 18)$ (q32; $\mathrm{q} 21)$ involving $B C L 2$ in follicular lymphoma, juxtaposes the MALT1 gene (also known as MLT and MLT1) next to the promoter region of the $\mathrm{Ig}$ heavy chain genes with subsequent MALT1 overexpression (7). The $t(11 ; 18)(\mathrm{q} 21 ; \mathrm{q} 21)$ translocation is the most common translocation, occurring in $15-40 \%$ of cases $(1,4,5,10,11)$. It results in the reciprocal fusion of cellular inhibitor of apoptosis protein 2 (cIAP2; also known as AIP1, API2, BIRC3, HAIP1, HIAP1, MALT2, and MIHC) on $11 \mathrm{q} 21$ with MALT1 on $18 \mathrm{q} 21$. The creation of a fusion protein encoded by CIAP2MALT1 on the derivative chromosome 11 is the pathogenetic event.

\section{The main players:}

\section{cIAP2, MALT1, and BCL10}

The cIAP2 protein belongs to the inhibitor of apoptosis protein (IAP) family, characterized by the presence of 1-3 bacu- loviral IAP repeat (BIR) domains (12-15). cIAP2 contains $3 \mathrm{~N}$-terminal BIRs, a middle caspase recruitment domain (CARD), and a C-terminal zinc-binding RING finger domain (Figure 1A). MALT1, a paracaspase, comprises an $\mathrm{N}$-terminal death domain (DD), followed by 2 Ig-like C2 domains, and a caspase-like domain (Figure 1B) (14-16). All the breakpoints in the cIAP2 gene occur downstream of the third BIR domain but upstream of the C-terminal RING domain, with over $90 \%$ of the breaks occurring just before the CARD (Figure 1A) $(1,2,4)$. Conversely, the breakpoints in MALT1 are variable but always upstream of the caspase-like domain (Figure 1B) $(1,2,4)$. Thus, the resulting fusion protein always compris- es the $\mathrm{N}$-terminal region of $C I A P 2$, with 3 intact BIR domains, and the C-terminal MALT1 region, containing an intact caspase-like domain (Figure 1C). The specific conservation of certain functional domains of CIAP2 and MALT1 to form a fusion product strongly suggests the importance and synergy of these domains in oncogenic activities. NF- $\kappa \mathrm{B}$ activation is one of the main downstream effects of the stimulation of cell-surface receptors, such as $\mathrm{B}$ cell or $\mathrm{T}$ cell receptors. In unstimulated cells, NF- $\mathrm{B}$ molecules are sequestered in the cytoplasm, because of the binding with inhibitory $\kappa \mathrm{B}(\mathrm{I} \kappa \mathrm{B})$ proteins. The IкB $\alpha$ protein is phosphorylated by the IкB kinase (IKK) heterodimer. The phosphorylation leads
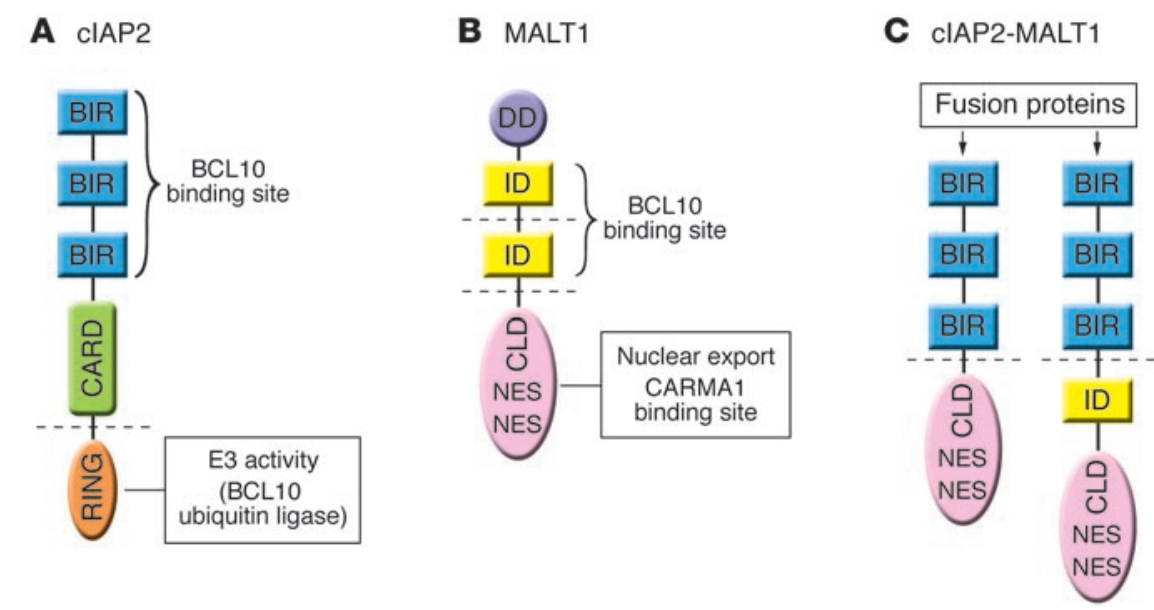

\section{Figure 1}

CIAP2, MALT1, and cIAP2-MALT1 organization. Schematic diagram showing the structure of clAP2 (A), MALT1 (B), and the 2 most commonly observed clAP2-MALT1 fusion proteins (C), including their known functional domains. The dashed lines show the most frequent breakpoint sites occurring in the $t(11 ; 18)(q 21 ; q 21)$ chromosomal translocation. The study by $\mathrm{Hu}$ et al. (22) in this issue of the $\mathrm{JCl}$ adds to our understanding of the properties of clAP2, showing BCL10 ubiquitin ligase activity in its $\mathrm{COOH}$-terminal region together with a BCL10 binding site within its $\mathrm{NH}_{2}$-terminal region. RING, really interesting new gene; ID, Ig-like domain; CLD, caspase-like domain. 
Step 1

Free ubiquitin is linked to a ubiquitin-activating enzyme (E1)

\section{t}

Step 2

The activated ubiquitin is transferred to a ubiquitinconjugating enzyme (E2)

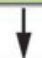

Step 3

The E2/ubiquitin complex is recruited to the target substrate by a ubiquitin ligase (E3)

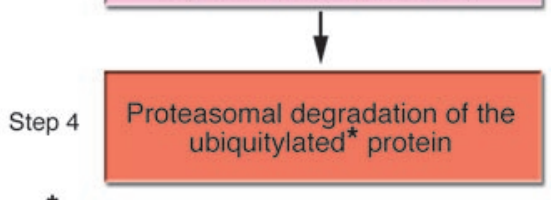

*Ubiquitylation does not always determine protein degradation

\section{Figure 2}

Schematic diagram showing the main steps of the ubiquitylation process leading to proteasomal degradation. It should be noted, however, that ubiquitylation does not always determine protein degradation (13).

to ubiquitylation and degradation of $\mathrm{I} \kappa \mathrm{B} \alpha$; NF- $\mathrm{B}$ can migrate to the nucleus and act as transcription factor. The IKK complex comprises 2 catalytically active kinases (IKK $\alpha$ and IKK $\beta$ ) and a regulatory component (IKK $\gamma$, also known as NEMO). Both MALT1 and BCL10, 2 of the genes involved in the above-mentioned translocations, are known to be upstream of the IKK complex (14-19). BCL10 binds to CARMA1 (also known as CARD11 and BIMP3) and to MALT1. The BCL10, CARMA1, and MALT1 complex activates NF-KB via IKK $\gamma$ degradation $(14-16,18,19)$. MALT1 binds to BCL10 at the Ig-like domains, and to CARMA1 at the caspase-like domain. The $\mathrm{t}(11 ; 18)(\mathrm{q} 21 ; \mathrm{q} 21)$ fusion protein cIAP2MALT1 is an activator of NF- $\kappa B$, and it bears a gain of function when compared with the WT MALT1 $(20,21)$.

\section{Novel insights into cIAP2 function}

The work presented by $\mathrm{Hu}$ et al. in this issue of the JCI (22) addresses a very important open question: the role of cIAP2 in normal mammalian cells and in the context of the fusion protein. Since the RING domain of IAPs can have a ubiquitin ligase (E3) activity, which determines the specificity of the ubiquitylation process, and since the latter is fundamental in protein degradation (Figure 2)
(13), Hu and colleagues hypothesized that cIAP2 might also be part of the antigen receptor signaling pathway. First, RNA and protein levels of BCL10 in mammalian cells were assessed in a series of $\mathrm{t}(11 ; 18)(\mathrm{q} 21 ; \mathrm{q} 21)$-positive and -negative cases. Indeed, protein and not RNA levels were higher in MALT lymphoma cases bearing the chromosomal translocation, suggesting a stabilization of the protein. The possible interaction between BCL10 and cIAP2 was then investigated by immunoprecipitation both in transfected human embryonic kidney 293T cells and in untransfected primary human $\mathrm{T}$ cells. BCL10 and cIAP2 clearly associated with each other in both experimental models. In particular, the association was increased after $\mathrm{T}$ cell activation and with the phosphorylated form of BCL10. By transfection of cIAP2 deletion mutants, BCL10 was shown to bind to the BIR region of CIAP2 and not to the RING domain or CARD. As is known for other IAPs (13), Hu et al. showed that the RING domain of cIAP2 has ubiquitin ligase (E3) activity (22). Because of the BIR-mediated binding, cIAP2 induced BCL10 ubiquitylation (Figure 3A). In T lymphocytes,
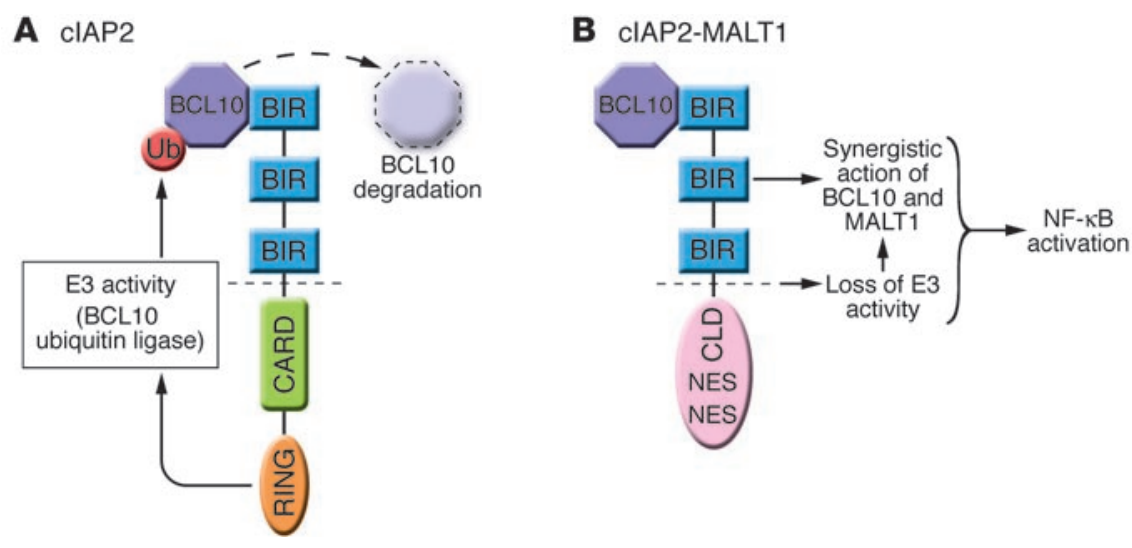

\section{Figure 3}

Loss of function and gain of function in CIAP2-MALT1. Schematic diagram showing the structure of WT clAP2 (A) and of the $t(11 ; 18)(q 21 ; q 21)$-associated clAP2-MALT1 fusion protein (B), as demonstrated in this issue of the $\mathrm{JCl}$ by Hu et al. (22). BCL10 can bind to the BIR region of WT clAP2. As shown by Hu et al., the RING domain of clAP2 has ubiquitin ligase (E3) activity. Because of BIR-mediated binding, cIAP2 leads to BCL10 ubiquitylation, a mechanism to regulate BCL10 activity after antigen receptor stimulation. The clAP2-MALT1 fusion protein created by the $t(11 ; 18)(q 21 ; q 21)$ chromosomal translocation always lacks the RING domain of cIAP2, while it always contains the BIR domains (B). The cIAP2-MALT1 protein can still bind BCL10 via the BIR domains, but it is not able to ubiquitylate it, because of the lack of the RING domain. The intact BCL10 synergistically increases clAP2-MALT1's intrinsic capacity for NF-KB activation. It is still not clear why the BIR-mediated BCL10 binding would not affect the transfer of BCL10 from the nucleus to the cytoplasm, which would be mediated, as recently shown (23), by the nuclear export signals (NESs), present in the cIAP2-MALT1 fusion protein. The dashed lines show the most frequent breakpoint sites occurring in the $t(11 ; 18)(q 21 ; q 21)$ chromosomal translocation. Ub, ubiquitin. 
expression of the cIAP2-MALT1 fusion protein. These data indicate that BCL10 expression might be needed for cIAP2MALT1 function. As a whole, the $\mathrm{Hu}$ et al. study (22) shows that cIAP2 as well as MALT1 and BCL10 is involved in physiologic antigen receptor stimulation. The EMZL-specific cIAP2-MALT1 fusion gene would behave both as a tumor suppressor gene, since it is no longer able to downregulate BCL10 expression, and also as an oncogene, since the fusion protein induces strong NF-KB activation (22). These data are in perfect accordance with the high expression levels of BCL10 observed in clinical EMZL cases bearing the $\mathrm{t}(11 ; 18)(\mathrm{q} 21 ; \mathrm{q} 21)$ translocation (9). The BCL10 protein is expressed only in the cytoplasm in non-neoplastic germinal center and marginal zone B cells as well as in EMZLs with neither $\mathrm{t}(1 ; 14)(\mathrm{p} 22 ; \mathrm{q} 32)$ nor $\mathrm{t}(11 ; 18)(\mathrm{q} 21 ; \mathrm{q} 21)$ translocations $(9)$. Conversely, nuclear expression is present both in the $t(1 ; 14)$-positive and in the $t(11 ; 18)$-positive EMZLs, suggesting that nuclear localization of BCL10 can occur as the result of 2 apparently independent cytogenetic events. While the significance of this change in cellular localization is not yet known, a possible partial explanation has recently been presented by Nakagawa et al. (23), albeit in partial disagreement with the data reported here by $\mathrm{Hu}$ et al. (22). Because of the presence of 2 nuclear export signal (NES) domains (Figure 1A), MALT1 would export BCL10 from the nucleus to the cytoplasm (23). In the presence of the $t(1 ; 14)(p 22 ; q 32)$ chromosomal translocation, the overexpression of BCL10 would lead to increased BCL10 nuclear compartmentalization (13). In the presence of the $t(14 ; 18)(q 32 ; q 21)$ translocation, with increased WT MALT1 expression, the localization of BCL10 would remain cytoplasmic, as observed in normal B cells $(23,24)$. In the presence of the $t(11 ; 18)(\mathrm{q} 21 ; \mathrm{q} 21)$ translocation, the effect on BCL10 cellular localization is still unclear. While in the study reported by Nakagawa et al. (23) the cIAP2-MALT1 fusion protein has lost the ability to bind BCL10, possibly explaining the accumulation of BCL10 in the nucleus, according to $\mathrm{Hu}$ and colleagues (22) this protein would retain its ability to bind BCL10. Since the constructs used in the 2 studies are relatively comparable, differences in the cell systems in which the experiments have been carried out could at least partially explain the differences in the observed results. However, further studies of the significance of nuclear BCL10 expression are mandatory.

\section{Outlook}

Another field where additional studies will be welcome is the role played by antigens and by the concomitant chronic phlogistic status in the pathogenesis of EMZL. Indeed, EMZLs are considered, at least partially, to be antigen dependent. In addition to epidemiological and therapeutic evidence, another basis for this belief was the demonstration that EMZL cells would often recognize self-antigens $(25,26)$. However, a very recent publication by Lenze et al. (27) reported new data disproving a direct role for the Igs expressed by EMZLs in sustaining lymphoma growth. The fact that only 1 out of 7 lymphomas expressed specific Igs (actually, directed against a plasma cell protein) makes the direct influence of antigen or self-antigen much less important in sustaining the growth of lymphoma cells. Indeed, in gastric MALT lymphoma, $H$. pylori-specific $\mathrm{T}$ cells are necessary to sustain the in vitro growth of lymphoma cells (28). Considering also the extreme importance of non-neoplastic cells in the pathogenesis and the definition of prognosis of follicular lymphomas (another indolent B cell lymphoma) $(29,30)$, further investigation of nonlymphoma cells in EMZL appears worthwhile.

The 4 recurrent chromosomal translocations demonstrate a site-specificity in terms of their incidence (10). Indeed, another open question that remains unaddressed is the possible effect of infectious agents in inducing individual translocations and how lymphoma risk depends on the microenvironmental effects of the interaction between genetic factors of the microorganisms (11) and the genetic background of the host (31). Nevertheless, if bacterial and host factors have a relevant impact on the initiation of the lymphomagenesis process, the role of the genetic damage eventually acquired by the tumor lymphocytes is important as well. In this context, the data provided by $\mathrm{Hu}$ et al. (22) shed light on the molecular consequences of the most common translocation in MALT lymphoma, showing that the $t(11 ; 18)(\mathrm{q} 21 ; \mathrm{q} 21)$ fusion product (cIAP2-MALT1) may concomitantly contribute to the lymphomagenesis, acting either as a tumor suppressor gene (loss of function) or as an oncogene (gain of function). Indeed, as a result of the cIAP2-MALT1 fusion, cIAP2's capacity for ubiquitylation and degradation of BCL10 is lost, and NF- $\mathrm{KB}$ appears to be synergistically activated by BCL10 (which is no longer degraded) and cIAP2-MALT1. The combination of both properties of cIAP2MALT1 appears crucial in the strong NF- $\kappa B$ activation observed in lymphocytes harboring the $\mathrm{t}(11 ; 18)(\mathrm{q} 21 ; \mathrm{q} 21)$ translocation, but the relative contribution to the oncogenic potential of these 2 properties of cIAP2MALT1 remains to be determined.

Address correspondence to: Emanuele Zucca, Head Lymphoma Unit, Oncology Institute of Southern Switzerland, Ospedale San Giovanni, 6500 Bellinzona, Switzerland. Phone: 41-91-811-8049; Fax: 41-91-811-9182; E-mail: emanuelezucca@ yahoo.com.

1. Isaacson, P.G., and Du, M.Q. 2004. MALT lymphoma: from morphology to molecules. Nat. Rev. Cancer. 4:644-653.

2. Zucca, E., and Bertoni, F. 2004. MALTlymphomas. Kluwer Academic. New York, New York, USA. 133 pp.

3. Bertoni, F., and Zucca, E. 2005. State-of-the-art therapeutics: marginal-zone lymphoma. J. Clin. Oncol. 23:6415-6420.

4. Farinha, P., and Gascoyne, R.D. 2005. Molecular pathogenesis of mucosa-associated lymphoid tissue lymphoma. J. Clin. Oncol. 23:6370-6378.

5. Dierlamm, J., et al. 1999. The apoptosis inhibitor gene API2 and a novel 18q gene, MLT, are recurrently rearranged in the $\mathrm{t}(11 ; 18)(\mathrm{q} 21 ; \mathrm{q} 21)$ associated with mucosa-associated lymphoid tissue lymphomas. Blood. 93:3601-3609.

6. Willis, T.G., et al. 1999. Bcl10 is involved in $\mathrm{t}(1 ; 14)(\mathrm{p} 22 ; \mathrm{q} 32)$ of MALT B cell lymphoma and mutated in multiple tumor types. Cell. 96:35-45.

7. Streubel, B., et al. 2002. T(14;18)(q32;q21) involving IGH and MALT1 is a frequent chromosomal aberration in MALT lymphoma. Blood. 101:2335-2339.

8. Streubel, B., Vinatzer, U., Lamprecht, A., Raderer, M., and Chott, A. 2005. T(3;14)(p14.1;q32) involving IGH and FOXP1 is a novel recurrent chromosomal aberration in MALT lymphoma. Leukemia. 19:652-658.

9. Ye, H., et al. 2000. BCL10 expression in normal and neoplastic lymphoid tissue: nuclear localization in MALT lymphoma. Am. J. Pathol. 157:1147-1154.

10. Murga Penas, E.M., et al. 2003. Translocations $\mathrm{t}(11 ; 18)(\mathrm{q} 21 ; \mathrm{q} 21)$ and $\mathrm{t}(14 ; 18)(\mathrm{q} 32 ; \mathrm{q} 21)$ are the main chromosomal abnormalities involving MLT/MALT1 in MALT lymphomas. Leukemia. 17:2225-2229.

11. Ye, H., et al. 2003. Variable frequencies of $\mathrm{t}(11 ; 18)(\mathrm{q} 21 ; \mathrm{q} 21)$ in MALT lymphomas of different sites: significant association with CagA strains of H. pylori in gastric MALT lymphoma. Blood. 102:1012-1018.

12. Reed, J.C. 2000. Mechanisms of apoptosis. Am. J. Pathol. 157:1415-1430.

13. Vaux, D.L., and Silke, J. 2005. IAPs, RINGs and ubiquitylation. Nat. Rev. Mol. Cell Biol. 6:287-297.

14. Lynch, O.T., and Gadina, M. 2004. Ubiquitination for activation: new directions in the NF-kappaB roadmap. Mol. Interv. 4:144-146.

15. Lucas, P.C., McAllister-Lucas, L.M., and Nunez, G. 2004. NF-kappaB signaling in lymphocytes: a new cast of characters. J. Cell Sci. 117:31-39.

16. Thome, M. 2004. CARMA1, BCL-10 and MALT1 in lymphocyte development and activation. Nat. Rev. Immunol. 4:348-359. 
17. Ho, L., et al. 2005. MALT1 and the API2-MALT1 fusion act between CD40 and IKK and confer NFkappa B-dependent proliferative advantage and resistance against FAS-induced cell death in B cells. Blood. 105:2891-2899.

18. Zhou, H., et al. 2004. Bcl10 activates the NF-kap$\mathrm{paB}$ pathway through ubiquitination of NEMO. Nature. 427:167-171.

19. Che, T., et al. 2004. MALT1/paracaspase is a signaling component downstream of CARMA1 and mediates T cell receptor-induced NF-kappaB activation. J. Biol. Chem. 279:15870-15876.

20. Lucas, P.C., et al. 2001. Bcl10 and MALT1, independent targets of chromosomal translocation in malt lymphoma, cooperate in a novel NF-kappa B signaling pathway. J. Biol. Chem. 276:19012-19019.

21. Zhou, H., Du, M.Q., and Dixit, V.M. 2005. Constitutive NF-kappaB activation by the $t(11 ; 18)(\mathrm{q} 21 ; \mathrm{q} 21)$ product in MALT lymphoma is linked to deregulated ubiquitin ligase activity. Cancer Cell. 7:425-431.
22. Hu, S., et al. 2006. cIAP2 is a ubiquitin protein ligase for BCL10 and is dysregulated in mucosaassociated lymphoid tissue lymphomas. J. Clin. Invest. 116:174-181. doi:10.1172/JCI25641.

23. Nakagawa, M., et al. 2005. MALT1 contains nuclear export signals and regulates cytoplasmic localization of BCL10. Blood. 106:4210-4216.

24. Ye, H., et al. 2005. MALT lymphoma with $\mathrm{t}(14 ; 18)(\mathrm{q} 32 ; \mathrm{q} 21) / \mathrm{IGH}-\mathrm{MALT} 1$ is characterized by strong cytoplasmic MALT1 and BCL10 expression. J. Pathol. 205:293-301.

25. Greiner, A., et al. 1994. Idiotype identity in a MALT-type lymphoma and B cells in Helicobacter pylori associated chronic gastritis. Lab. Invest. 70:572-578.

26. Hussell, T., Isaacson, P.G., Crabtree, J.E., Dogan, A., and Spencer, J. 1993. Immunoglobulin specificity of low grade B cell gastrointestinal lymphoma of mucosa-associated lymphoid tissue (MALT) type. Am. J. Pathol. 142:285-292.
27. Lenze, D., et al. 2005. Influence of antigen on the development of MALT-lymphoma. Blood. Online publication ahead of print.

28. Hussell, T., Isaacson, P.G., Crabtree, J.E., and Spencer, J. 1996. Helicobacter pylori-specific tumourinfiltrating $\mathrm{T}$ cells provide contact dependent help for the growth of malignant B cells in low-grade gastric lymphoma of mucosa-associated lymphoid tissue. J. Pathol. 178:122-127.

29. Dave, S.S., et al. 2004. Prediction of survival in follicular lymphoma based on molecular features of tumor-infiltrating immune cells. N. Engl. J. Med. 351:2159-2169.

30. de Jong, D. 2005. Molecular pathogenesis of follicular lymphoma: a cross talk of genetic and immunologic factors. J. Clin. Oncol. 23:6358-6363.

31. Rollinson, S., et al. 2003. Gastric marginal zone lymphoma is associated with polymorphisms in genes involved in inflammatory response and antioxidative capacity. Blood. 102:1007-1011.

\title{
Serum peptidome for cancer detection: spinning biologic trash into diagnostic gold
}

\author{
Lance A. Liotta and Emanuel F. Petricoin
}

Center for Applied Proteomics and Molecular Medicine, College of Arts and Sciences, George Mason University, Manassas, Virginia, USA.

\begin{abstract}
The low molecular weight region of the serum peptidome contains protein fragments derived from 2 sources: (a) high-abundance endogenous circulating proteins and (b) cell and tissue proteins. While some researchers have dismissed the serum peptidome as biological trash, recent work using mass spectrometry-based (MS-based) profiling has indicated that the peptidome may reflect biological events and contain diagnostic biomarkers. In this issue of the JCI, Villanueva et al. report on MS-based peptide profiling of serum samples from patients with advanced prostate, bladder, or breast cancer as well as from healthy controls (see the related article beginning on page 271). Surprisingly, the peptides identified as cancer-type-specific markers proved to be products of enzymatic breakdown generated after patient blood collection. The impact of these results on cancer biomarker discovery efforts is significant because it is widely believed that proteolysis occurring ex vivo should be suppressed because it destroys endogenous biomarkers. Villanueva et al. now suggest that this suppression may in fact be preventing biomarker generation.
\end{abstract}

\section{The illusion of a dry biomarker pipeline}

Despite the tremendous urgency to identify clinically useful biomarkers for early

Nonstandard abbreviations used: BRCA2, breast cancer 2, early onset; LMW, low molecular weight; MALDITOF, matrix-assisted laser desorption/ionization-timeof-flight; MS, mass spectrometric, mass spectrometry.

Conflict of interest: The authors have no personal conflicts, financial or otherwise. They have US government-assigned and -owned patent applications that cover aspects of serum biomarker discovery and amplification discussed in this manuscript. As former government employees, if the US government licenses these patents the authors are entitled to receive a share of the royalties.

Citation for this article: J. Clin. Invest. 116:26-30 (2006). doi:10.1172/JCI27467. disease detection, there have been only a few recent examples of such analytes that have had any real impact at the bedside $(1,2)$. Many scientists have pointed to what they perceive to be a dried-up blood-borne cancer biomarker pipeline for disease detection since recent searches for a single, cancer-specific marker have not proved fruitful. In response to this challenge, investigators in the field of proteomics have shifted their focus in an effort to utilize experimental methods such as mass spectrometry (MS), which does not require knowledge of a protein's amino acid sequence prior to effective detection of the analyte. These MS-based methods offer new approaches whereby signatures of multiple analytes measured simultaneously comprise the diagnostic classifier (3-10). MS analysis of blood proteome is proving facile at probing and profiling proteomic information that may encompass hundreds of candidate disease biomarkers without the need for a priori knowledge of their existence or relevance to disease states (4-10).

Within this field of research, interest continues to grow regarding a previously unexplored reservoir - the array of existing proteins in a patient's serum (coined as the serum proteome), particularly those of low molecular weight (LMW), as well as the metabolic products of these serum proteins (the serum peptidome, fragmentome, or degradome) $(11,12)$. Prior efforts in the search for serum and plasma protein biomarkers utilized gel-based separation technologies, which cannot readily separate and distinguish molecules of less than $10 \mathrm{kDa}$ in size. In contrast, MS is particularly well suited for the detection of molecules within the LMW range of analysis ( $<20 \mathrm{kDa})$. In recognition of this attribute, investigators began to use MS to explore the LMW component of the circulatory proteome in order to determine whether the LMW pool contained any disease-related biomarker candidates. This method was first applied to examine the sera of patients with ovarian cancer (4) and then later for other cancers (5-9) 